\title{
Representing Group Codes as Permutation Codes
}

\author{
Ezio Biglieri ${ }^{\dagger}$ \\ John K. Karlof* \\ Emanuele Viterbo ${ }^{\dagger}$
}

November 19, 2001

\begin{abstract}
Given an abstract group $\mathcal{G}$, an $N$ dimensional orthogonal matrix representation $\mathbf{G}$ of $\mathcal{G}$, and an "initial vector" $\mathbf{x} \in \mathbf{R}^{N}$, Slepian defined the group code generated by the representation $\mathbf{G}$ to be the set of vectors $\mathbf{G x}$. If $\mathbf{G}$ is a group of permutation matrices, the set $\mathbf{G x}$ is called a "permutation code". For permutation codes a 'stack algorithm' decoder exists that, in the presence of low noise, produces the maximum-likelihood estimate of the transmitted vector by using far fewer computations than the standard decoder. In this paper a new concept of equivalence of codes of different dimensions is presented which is weaker than the usual definition of equivalent codes. We show that every group code is (weakly) equivalent to a permutation code and we discuss the minimal degree of this permutation code.
\end{abstract}

*J. Karlof is with the Mathematical Sciences Department • University of North Carolina • Wilmington, NC 28403

${ }^{\dagger}$ E. Biglieri and E. Viterbo are with Dipartimento di Elettronica • Politecnico di Torino • I-10129 Torino (Italy) Their contribution to this research was sponsored by CNR under "Progetto Finalizzato Trasporti." 


\section{Introduction}

Group codes, as defined by Slepian (see [10] and references therein) are defined as follows. ${ }^{1}$ Consider a group $\mathbf{G}$ of $N \times N$ orthogonal matrices which forms an injective representation of an abstract group $\mathcal{G}$ with $M$ elements, and an "initial vector" $\mathbf{x} \in \mathbf{R}^{N}, \mathbf{R}^{N}$ the Euclidean $N$-dimensional space. A group code $\mathcal{X}$ is the orbit of $\mathbf{x}$ under $\mathcal{G}$, i.e., the set of vectors $\mathbf{G x}$. By assuming that the only solution of the equation $G \mathbf{x}=\mathbf{x}, G \in \mathbf{G}$, is $G=I$ (the identity matrix), the code $\mathcal{X}$ has $M$ elements. We say $\mathcal{X}$ is an $[M, N]$ group code and denote $\mathbf{x}_{g}$ the code vector associated with $g \in \mathcal{G}$.

When a codeword $\mathbf{x}_{g}$ of $\mathcal{X}$ is transmitted over the additive white Gaussian noise channel, the optimum (i.e., maximum-likelihood) decoder, upon receiving the noisy vector $\mathbf{r}=\mathbf{x}_{g}+\mathbf{n}$, chooses as the most likely transmitted vector the one that yields

$$
\min _{h \in \mathcal{G}}\left\|\mathbf{r}-\mathbf{x}_{h}\right\|^{2}
$$

If $\mathcal{G}$ is not endowed with any special structure, decoding (i.e., the solution of (1)) is obtained by an exhaustive search among all the candidates $g \in \mathcal{G}$. This requires a number of calculations $\nu_{C}=N M$ (in fact, $M$ scalar products of $N$ terms each must be computed) and a storage of $\nu_{S}=N M$ real numbers ( $M$ vectors of $N$ components each). Define the number of bits per dimension carried by the constellation as

$$
r=\frac{\log _{2} M}{N}
$$

then we have $\nu_{C}=\nu_{S}=N 2^{r N}$, which shows that the complexity of the decoder grows exponentially with the number of dimensions and with the number of bits per dimension. A permutation code is a group code obtained by applying to the initial vector $\mathbf{x}$ a group $\mathbf{G}$ of permutations (i.e. $\mathbf{G}$ is a group of permutation matrices). If $\mathcal{X}$ is a permutation code, then a less complex decoder that is equivalent to maximum likelihood is available.

Slepian [9] has studied permutation codes with $\mathcal{G}$ the full symmetric group $\mathcal{S}_{n}$. In this case a very simple decoder exists that is equivalent to maximum likelihood. Karlof [4] has described a "stack algorithm" decoder for arbitrary permutation codes that, in the presence of low noise, produces

\footnotetext{
${ }^{1}$ The reader is warned that the term "group code" is being used of late with a different meaning, i.e., to denote block or convolutional codes defined over an alphabet forming a group. Accordingly, some authors use a different term (e.g., "orbit codes" [8]) instead of Slepian's "group codes."). We use here the original definition to be consistent with our references.
} 
the maximum-likelihood vector using fewer calculations than the standard maximum-likelihood decoder.

Two $[M, N]$ codes $\mathcal{X}_{1}$ and $\mathcal{X}_{2}$ are defined to be equivalent if there exists an orthogonal $N$ by $N$ matrix $O$ such that $O \mathcal{X}_{1}=\mathcal{X}_{2}$. Equivalent codes have congruent Voronoi regions and thus have the same error performance over the Gaussian channel. We extend the definition of equivalence to codes in different dimensions with the same number of elements. In this case, we say the two codes are equivalent if they have the same configuration matrix, i.e., the Gram matrix of their scalar products. Then the two codes have the same set of distances between codewords as in the case of equivalent codes of the same dimension. We note that this definition is weaker than the usual definition since the codes are not, in general, orthogonal transformations of each other. It what follows it should be clear from the context which definition of equivalence is being used.

In this paper, using the fact that every group is isomorphic to a permutation group, we find the minimum degree of this permutation group, show that every group code is (weakly) equivalent to a permutation code, and describe how to find the minimum degree of the equivalent permutation code.

\section{Finding an equivalent permutation code}

Let $\mathcal{G}$ be a group. A permutation representation of degree $n$ [6, Chap. 7] of $\mathcal{G}$ is a homomorphism of $\mathcal{G}$ into $\mathcal{S}_{n}$, or the image of $\mathcal{G}$ under the homomorphism. If the homomorphism is an isomorphism, we say that the representation is faithful.

In general, every group $\mathcal{G}$ with order $|\mathcal{G}|$ is isomorphic to a subgroup of $\mathcal{S}_{|\mathcal{G}|}$. Let $\mathcal{H}$ denote a subgroup of $\mathcal{G}$ and let $\mathcal{R}$ be the set of right cosets of $\mathcal{H}$ in $\mathcal{G}$. Then

$$
\mathcal{G}=\bigcup_{\mathcal{H} r \in \mathcal{R}} \mathcal{H} r
$$

is the decomposition of $\mathcal{G}$ into right cosets of $\mathcal{H}$. To every $g \in \mathcal{G}$ assign the permutation

$$
\pi_{g}: \mathcal{R} \rightarrow \mathcal{R} \text { where } \pi_{g}(\mathcal{H} r)=\mathcal{H} r g
$$

The set $\Gamma=\left\{\pi_{g} \mid g \in \mathcal{G}\right\}$ is a transitive permutation group of degree $n=|\mathcal{G}| /|\mathcal{H}|$ and is the permutation representation of $\mathcal{G}$ induced by $\mathcal{H}$ [6]. Every transitive permutation representation of $\mathcal{G}$ can be obtained in this way. When $\mathcal{H}=\{e\}$, the identity of $\mathcal{G}$, the representation induced by $\mathcal{H}$ 
is called the right regular representation of $\mathcal{G}$. The left regular representation can be defined in a similar way.

The minimum $n$ corresponds to the maximum $|\mathcal{H}|$ such that the representation $\Gamma$ is faithful, i.e., such that the kernel of the homomorphism of $\mathcal{G}$ onto $\Gamma$ is the identity. This kernel can be characterized as the maximal normal subgroup of $\mathcal{G}$ contained in $\mathcal{H}$ [6, Chap. 7]. Consequently, if $\mathcal{H}^{\prime}$ denotes the largest non-normal subgroup of $\mathcal{G}$ that does not include normal subgroups of $\mathcal{G}$ other than the identity, then $n$ is given by the ratio

$$
n=\frac{|\mathcal{G}|}{\left|\mathcal{H}^{\prime}\right|}
$$

Example 2.1 Icosahedral group. [7, p. 32] Let $\mathcal{G}=<x, y, z \mid x^{3}=y^{2}=z^{2}=(x y)^{3}=(y z)^{3}=$ $(x z)^{2}=1>$. Then $\mathcal{G}$ is a simple group of order 60 . Let $\mathcal{H}=<x, y>$. Then, the order of $\mathcal{H}$ is 12 and since $\mathcal{G}$ has no subgroups of order larger than 12, $\mathcal{H}$ is the largest non-normal subgroup of $\mathcal{G}$ that does not contain any non-trivial normal subgroups of $\mathcal{G}$. Thus, $\mathcal{G}$ is isomorphic to a permutation group, $\Gamma_{\mathcal{H}}$, of degree 5 and of order 60 . The set of right cosets is $\mathcal{R}=\left\{\mathcal{H}, \mathcal{H} z, \mathcal{H} z y, \mathcal{H} z y x, \mathcal{H} z y x^{2}\right\}$ and $\Gamma_{\mathcal{H}}=<(3,4,5),(2,3)(4,5),(1,2)(4,5)>$. For example, $\pi_{z}=(1,2)(4,5)$ since

$$
\begin{aligned}
\pi_{z}(\mathcal{H}) & =\mathcal{H} z \\
\pi_{z}(\mathcal{H} z) & =\mathcal{H} z^{2}=\mathcal{H} \\
\pi_{z}(\mathcal{H} z y) & =\mathcal{H} z y z=\mathcal{H} y z y=\mathcal{H} z y \\
\pi_{z}(\mathcal{H} z y x) & =\mathcal{H} z y x z=\mathcal{H} z y z x^{2}=\mathcal{H} y z y x^{2}=\mathcal{H} z y x^{2} \\
\pi_{z}\left(\mathcal{H} z y x^{2}\right) & =\mathcal{H} z y x^{2} z=\mathcal{H} z y x z x^{2}=\mathcal{H} z y z x=\mathcal{H} z y x .
\end{aligned}
$$

Now if $\mathcal{G}$ is abelian or a Sylow $p$-group then all its subgroups are normal. So $\left|\mathcal{H}^{\prime}\right|=1$, and hence $n=|\mathcal{G}|$. If $\mathcal{G}=\mathcal{S}_{m}, m=3$ or $m \geq 5$, then its only non-trivial normal subgroup is the alternating group $\mathcal{A}_{m}$, while $\mathcal{G}$ does admit the subgroup $\mathcal{S}_{m-1}$. Hence

$$
n=\frac{\left|\mathcal{S}_{m}\right|}{\left|\mathcal{S}_{m-1}\right|}=m \text {. }
$$

Thus the closer $\mathcal{G}$ is to an abelian group, the larger is the value of $n$. 
Theorem 2.1 Suppose $\mathcal{G}$ is a finite abstract group with irreducible real characters $\chi_{1}, \chi_{2}, \ldots, \chi_{p}$. Consider a faithful representation $\rho: \mathcal{G} \rightarrow \mathbf{G}$ where $\mathbf{G}$ is a group of orthogonal $N \times N$ matrices. Let $\chi_{\rho}$ be the character of $\rho$ and suppose $\chi_{\rho}=\sum_{i=1}^{p} a_{i} \chi_{i}$. Let $\mathbf{x} \in \mathbf{R}^{N}$ and form the group code $\mathcal{X}=\mathbf{G} \mathbf{x}=\{\rho(g) \mathbf{x}: g \in \mathcal{G}\}$. Suppose $\mathcal{H}$ is a subgroup of $\mathcal{G}$ and form the permutation representation $\phi: \mathcal{G} \rightarrow \Gamma=\left\{\pi_{g} \mid g \in \mathcal{G}\right\}$ induced by $\mathcal{H}$. Let $\chi_{\phi}$ be the character of $\phi$ and suppose $\chi_{\phi}=\sum_{i=1}^{p} b_{i} \chi_{i}$. If $\phi$ is faithful and $b_{i} \geq a_{i} \forall i$, then $\Gamma$ generates a permutation code equivalent to $\mathcal{X}$.

Proof: Let $\psi_{i}$ be the irreducible representation of $\mathcal{G}$ afforded by $\chi_{i}$. Without loss of generality assume that $a_{i} \neq 0$ for $1 \leq i \leq m$ and $a_{i}=0$ for $i>m$. Then there exists an orthogonal $N \times N$ matrix $U$ such that $U \rho(g) U^{T}=\oplus_{i=1}^{m} a_{i} \psi_{i}(g), \forall g \in \mathcal{G}$ (i.e. $\rho(g)$ is equivalent to the direct sum of $a_{1}$ copies of $\psi_{1}(g) \ldots a_{m}$ copies of $\left.\psi_{m}(g)\right)$. Let $\bar{x}=U x$. Then $\mathcal{X}$ and $\left\{\left(\oplus_{i=1}^{m} a_{i} \psi_{i}(g)\right) \bar{x} \mid g \in \mathcal{G}\right\}$ are equivalent $N$ dimensional codes.

We will consider $\Gamma$ as a group of $n \times n$ permutation matrices where $n=\frac{|\mathcal{G}|}{|\mathcal{H}|}$. Then there exists an orthogonal $n \times n$ matrix $V$ such that $V \phi(g) V^{T}=\oplus_{i=1}^{m} a_{i} \psi_{i}(g) \oplus_{i=1}^{m}\left(b_{i}-a_{i}\right) \psi_{i}(g) \oplus_{i=m+1}^{p} b_{i} \psi_{i}(g), \forall g \in$ $\mathcal{G}$.

Let $x^{0}$ be a zero padded $n$ dimensional version of $\bar{x}$ (i.e. $\left.x^{0}=\left(\bar{x}^{T}, 0 \ldots 0\right)^{T}\right)$. Then $\left\{\left(\oplus_{i=1}^{p} b_{i} \psi_{i}(g)\right)\right.$ and $\left\{\phi(g)\left(V^{T} x^{0}\right)\right\}$ are equivalent $n$ dimensional codes. Clearly the $N$ dimensional code $\left\{\oplus_{i=1}^{m} a_{i} \psi_{i}(g)\right.$ and the $n$ dimensional code $\left\{\oplus_{i=1}^{p} b_{i} \psi_{i}(g) x^{0}\right\}$ have the same number of elements and the same configuration matrix. Thus $\Gamma$ generates a permutation code equivalent to $\mathcal{X}$.

Corollary 2.1 Every group code is equivalent to at least one permutation code.

Proof: Let $\mathcal{H}=\{e\}$. Then $\phi$ is the right regular permutation representation of $\mathcal{G}, \phi(g)=$ $\oplus_{i=1}^{p} b_{i} \psi_{i}(g)$ where $b_{i}=\operatorname{deg}\left(\psi_{i}\right)$ if $\psi_{i}$ is also irreducible over the complex field (i.e. $\psi_{i}$ is a complex irreducible representation of the first kind) and $b_{i}=\frac{1}{2}$ or $\frac{1}{4}$ times $\operatorname{deg}\left(\psi_{i}\right)$ otherwise. If $a_{i}>b_{i}$, then more copies of $\phi$ may be used.

Example 2.2 The 4-PSK signal set can be generated by the following representation of the cyclic group, $\mathcal{G}$, of order four

$$
\left[\begin{array}{cc}
0 & 1 \\
-1 & 0
\end{array}\right]\left[\begin{array}{cc}
-1 & 0 \\
0 & -1
\end{array}\right]\left[\begin{array}{cc}
0 & -1 \\
1 & 0
\end{array}\right]\left[\begin{array}{ll}
1 & 0 \\
0 & 1
\end{array}\right]
$$


using the initial vector $\mathbf{x}=[1,0]^{T}$. The irreducible real representations of this cyclic group are the representation above, denoted $\psi_{2}(g)$, the identity representation $\psi_{1}(g)$, associating +1 with all the groups elements, and the alternating representation $\psi_{-1}(g)$, associating -1 with the first and third elements of $\mathcal{G}$ and +1 with the second and fourth elements. Consequently, letting $\phi$ denote the right regular representation, an orthogonal matrix, $V$, exists such that

$$
\psi_{1}(g) \oplus \psi_{-1}(g) \oplus \psi_{2}(g)=V \phi(g) V^{T} .
$$

The matrix $V$ is found in [1]:

$$
V=\frac{1}{2}\left[\begin{array}{cccc}
1 & 1 & 1 & 1 \\
1 & -1 & 1 & -1 \\
\sqrt{2} & 0 & -\sqrt{2} & 0 \\
0 & -\sqrt{2} & 0 & \sqrt{2}
\end{array}\right]
$$

By applying $V^{T}$ to the zero-padded version of $\mathbf{x}, \mathbf{x}^{0}=[0,0,1,0]^{T}$, we get the initial vector of the permutation code equivalent to 4 -PSK: $[\sqrt{2} / 2,0,-\sqrt{2} / 2,0]^{T}$.

In practice, it is often difficult to find the matrices $U$ and $V$ in the proof of the previous theorem. Also, the degree $n$ of the permutation representation may be prohibitively large. The procedure is greatly simplified in the case that the image of $\phi$ is doubly transitive.

Corollary 2.2 Suppose $\Gamma$ is doubly transitive. Then

1. $\rho$ is irreducible,

2. $\phi=1 \oplus \rho$, (here, we use 1 to denote the identity representation of $\mathcal{G})$

3. $n=N+1$,

4. $U$ is the identity matrix, and

5. V may be taken to be

$$
\left[\begin{array}{cccccc}
\gamma & \gamma & \gamma & \ldots & \gamma & \gamma \\
\beta_{1} & -\gamma_{1} & -\gamma_{1} & \ldots & -\gamma_{1} & -\gamma_{1} \\
0 & \beta_{2} & -\gamma_{2} & \ldots & -\gamma_{2} & -\gamma_{2} \\
\cdot & \cdot & \cdot & . & \cdot & \cdot \\
0 & 0 & 0 & \ldots & \beta_{n-1} & -\beta_{n-1}
\end{array}\right]
$$


where $n \gamma^{2}=1,(n-j) \gamma_{j}^{2}+\beta_{j}^{2}=1, \beta_{j}-(n-j) \gamma_{j}=0$.

Proof: It is well known [3, p. 230] that a doubly transitive permutation representation may be written as the direct sum of the identity representation and an irreducible representation. The matrix $V$ is given in [2].

Given an irreducible representation $\rho: \mathcal{G}: \rightarrow \mathbf{G}$, a method to find an appropriate $\mathcal{H}$ is to use a computer algebra system such as MAGMA to print out all subgroups of $\mathcal{G}$ of low index and then, if necessary, use the characters of $\mathcal{G}$ to find which of the induced permutation representations contain $\rho$. This is illustrated in the following example.

Example 2.3 Let $\mathcal{G}=$ the icosahedral group. This group has four nontrivial irreducible orthogonal representations[7, p. 313], two of degree 3, one of degree 4, and one of degree 5. We label these $\rho_{1}, \rho_{2}, \rho_{3}, \rho_{4}$ and their characters $\chi_{1}, \chi_{2}, \chi_{3}, \chi_{4}$ respectively. We use MAGMA to find the low index subgroups of $\mathcal{G}$. There are four of index 12 or less. They are $\mathcal{H}$ of index 5 from example 2.1, $\mathcal{I}=<y, z x^{2}>$ of index $6, \mathcal{K}=<x, z>$ of index 10 , and $\mathcal{L}=<x y z>$ of index 12. Denote the induced permutation representations by $\phi_{\mathcal{H}}, \phi_{\mathcal{I}}, \phi_{\mathcal{K}}$ and $\phi_{\mathcal{L}}$ respectively. Only $\phi_{\mathcal{H}}$ and $\phi_{\mathcal{I}}$ are doubly transitive. Thus $\phi_{\mathcal{H}}=1 \oplus \rho_{3}$ and $\phi_{\mathcal{I}}=1 \oplus \rho_{4}$ and by corollary 2.2 any group codes generated by $\rho_{3}$ or $\rho_{4}$ can be easily represented by equivalent permutation codes.

To find permutation codes equivalent to group codes generated by $\rho_{1}$ or $\rho_{2}$, we investigate the images and characters of $\phi_{\mathcal{K}}$ and $\phi_{\mathcal{L}}$ which we denote by $\Gamma_{\mathcal{K}}, \Gamma_{\mathcal{L}}, \chi_{\mathcal{K}}$, and $\chi_{\mathcal{L}}$ respectively. The orders of the five conjugacy classes of $\mathcal{G}$ are $\left|C_{1}\right|=1,\left|C_{2}\right|=12,\left|C_{3}\right|=12,\left|C_{4}\right|=15$, and $\left|C_{5}\right|=20$. The representatives of the corresponding conjugacy classes of $\Gamma_{\mathcal{K}}$ and $\Gamma_{\mathcal{L}}$ are $\{(1),(1,2,8,10,6)(3,9,7,5,4),(1,8,6,2,10)(3,7,4,9,5),(1,2)(3,5)(6,8)(7,9),(2,3,5)(4,7,6)(8,10$, and $\{(1),(2,11,8,5,7)(3,10,12,4,9),(2,8,7,11,5)(3,12,9,10,4),(1,3)(2,4)(5,11)(6,8)(7,12)(9,10)$ $(1,2,5)(3,7,9)(4,10,6)(8,12,11)\}$ respectively. The character table of $\mathcal{G}$ is :

\begin{tabular}{c|ccccc} 
& $C_{1}$ & $C_{2}$ & $C_{3}$ & $C_{4}$ & $C_{5}$ \\
\hline 1 & 1 & 1 & 1 & 1 & 1 \\
$\chi_{1}$ & 3 & $\frac{1+\sqrt{5}}{2}$ & $\frac{1-\sqrt{5}}{2}$ & -1 & 0 \\
$\chi_{2}$ & 3 & $\frac{1-\sqrt{5}}{2}$ & $\frac{1+\sqrt{5}}{2}$ & -1 & 0 \\
$\chi_{3}$ & 4 & -1 & -1 & 0 & 1 \\
$\chi_{4}$ & 5 & 0 & 0 & 1 & -1.
\end{tabular}


Since $\chi_{\mathcal{K}}(g)$, and $\chi_{\mathcal{L}}(g)$ equal the number of elements $\phi_{\mathcal{K}}(g)$ and $\phi_{\mathcal{L}}(g)$ fix the following character inner products are easily computed,

$$
\begin{aligned}
1 & =\frac{1}{|\mathcal{G}|} \sum_{g \in \mathcal{G}} \chi_{\mathcal{K}}(g) \chi_{3}(g) \\
& =\frac{1}{|\mathcal{G}|} \sum_{g \in \mathcal{G}} \chi_{\mathcal{K}}(g) \chi_{4}(g) \\
1 & =\frac{1}{|\mathcal{G}|} \sum_{g \in \mathcal{G}} \chi_{\mathcal{L}}(g) \chi_{1}(g) \\
& =\frac{1}{|\mathcal{G}|} \sum_{g \in \mathcal{G}} \chi_{\mathcal{L}}(g) \chi_{2}(g) \\
& =\frac{1}{|\mathcal{G}|} \sum_{g \in \mathcal{G}} \chi_{\mathcal{L}}(g) \chi_{4}(g) .
\end{aligned}
$$

Now, since every permutation representation contains the identity representation we have, $\rho_{\mathcal{K}}=$ $1 \oplus \rho_{3} \oplus \rho_{4}$ and $\rho_{\mathcal{L}}=1 \oplus \rho_{1} \oplus \rho_{2} \oplus \rho_{4}$. So we can represent group codes generated by $\rho_{1}$ and $\rho_{2}$ by equivalent permutation codes but the degree would be 12 and the matrix $V$ would have to be found.

We conclude with an example which summarizes the main result of the paper.

Example 2.4 Let $\mathcal{G}=$ the icosahedral group. Consider the 4-dimensional group code $\mathcal{X}=\left\{\rho_{3}(g) \mathbf{x}\right.$ : $g \in \mathcal{G}\}$. The image of $\rho_{3}$ can be found in [7, p. 313]. We use a modification of the algorithm in [5] to find the optimal initial vector $\mathbf{x}=[-0.68222,-0.49471,-0.44657,-0.30070]$ for this representation. The minimum squared Euclidean distance is $d_{m i n}^{2}=0.447056$. We are then under the hypothesis of Corollary 2.1. We use the degree 5 permutation representation $\phi_{\mathcal{H}}=1 \oplus \rho_{3}$ and transform the zero padded vector $\mathbf{x}^{0}=[0,-0.68222,-0.49471,-0.44657,-0.30070]$ to the initial vector $V^{T} \mathbf{x}^{0}=[-0.61010,-0.27588,-0.06926,0.26504,0.69020]$ for the equivalent permutation code generated by $\Gamma_{\mathcal{H}}$.

We finally note that in practice the code is transmitted over the AWGN channel using the low-dimensional constellation in order to save on the spectral efficiency. The received vector $\mathbf{r}$ is first zero-padded as for the initial vector and then transformed into $\mathbf{y}=V^{T} \mathbf{r}^{0}$. Now, $\mathbf{y}$ can be ML decoded with the permutation code decoder. We note that this is an orthogonal transformation on the received vector which does not modify the additive noise statistics. In the above example 
the operation is particularly convenient since the code dimension is only increased by one. On the other hand, if we wanted to use the 3 -dimensional codes generated by the representations $\rho_{1}$ or $\rho_{2}$ we would need to use a degree 12 permutation representation.

\section{References}

[1] E. Biglieri and M. Elia, "Cyclic-group codes for the Gaussian channel," IEEE Trans. Inform. Theory, Vol. IT-22, pp. 624-629, September 1976.

[2] I. F. Blake, "Distance properties of group codes for the Gaussian channel," SIAM J. Appl. Math., Vol. 23, No. 3, pp. 312-324, November 1972.

[3] C. W. Curtis and I. Reiner, Representation Theory of Finite Groups and Associative Algebras, Interscience, New York, 1962.

[4] J. K. Karlof, "Decoding spherical codes for the Gaussian channel," IEEE Trans. Inform. Theory, Vol. 39, No. 1, pp. 60-65, January 1993.

[5] J. K. Karlof, "Permutation codes for the Gaussian channel," IEEE Trans. Inform. Theory, Vol. 35, No. 4, pp. 726-732, July 1989.

[6] R. Kochendörffer, Group Theory. London: McGraw-Hill, 1965.

[7] J. S. Lomont, Applications of Finite Groups, Academic Press, New York, 1959.

[8] V. M. Sidelnikov, "On a finite group of matrices generating orbit codes on Euclidean sphere," Proc. $199^{7}$ IEEE Intern. Symp. Inform. Theory, Ulm, Germany, p. 436, June 29-July 4, 1997.

[9] D. Slepian, "Permutation modulation," IEEE Proc., pp. 228-236, March 1965.

[10] D. Slepian, "Group codes for the Gaussian channel," Bell System Technical Journal, Vol. 47, pp. 575-602, April 1968. 